\title{
B-Mode Ultrasonography versus Contrast-Enhanced Ultrasonography for Surveillance of Hepatocellular Carcinoma: A Prospective Multicenter Randomized Controlled Trial
}

\author{
Masatoshi Kudo $^{a}$ Kazuomi Ueshima $^{a}$ Yukio Osaki ${ }^{b}$ Masashi Hirookac \\ Yasuharu Imai $^{d}$ Kazunobu Aso ${ }^{e}$ Kazushi Numata ${ }^{f}$ Masayuki Kitano ${ }^{g}$

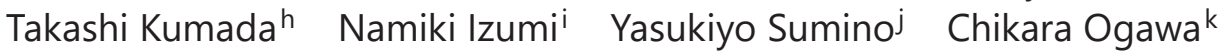 \\ Kohei Akazawal for the SELECTED Study Group, Japan \\ a Department of Gastroenterology and Hepatology, Kindai University School of Medicine, \\ Osaka, Japan; ${ }^{b}$ Department of Gastroenterology and Hepatology, Osaka Red Cross \\ Hospital, Osaka, Japan; ' Department of Gastroenterology and Metabology, Ehime \\ University Graduate School of Medicine, Ehime, Japan; dDepartment of Gastroenterology, \\ Ikeda Municipal Hospital, Osaka, Japan; ${ }^{e}$ Division of Metabolism and Biosystemic \\ Science, Department of Internal Medicine, Asahikawa Medical College, Asahikawa, Japan; \\ ${ }^{f}$ Gastroenterological Center, Yokohama City University Medical Center, Yokohama, Japan; \\ gSecond Department of Internal Medicine, Wakayama Medical University, Wakayama, \\ Japan; h Department of Gastroenterology and Hepatology, Ogaki Municipal Hospital, Ogaki, \\ Japan; 'Department of Gastroenterology and Hepatology, Musashino Red Cross Hospital, \\ Musashino, Japan; jDivision of Gastroenterology and Hepatology, Department of Internal \\ Medicine, Toho University Medical Center, Tokyo, Japan; ' Department of Gastroenterology \\ and Hepatology, Takamatsu Red Cross Hospital, Takamatsu, Japan; 'Department of Medical \\ Informatics, Niigata University Medical and Dental Hospital, Niigata City, Japan
}

\section{Keywords}

B-mode ultrasonography · Contrast-enhanced ultrasound · Hepatocellular carcinoma · Liver cirrhosis · Sonazoid

\begin{abstract}
Background: Current practice guidelines recommend the use of ultrasound (US) as an initial surveillance tool for hepatocellular carcinoma (HCC) in patients with liver cirrhosis. Patients with liver cirrhosis, however, frequently have coarse liver parenchyma, masking the presence of tiny nodules during B-mode US. Contrast-enhanced US (CEUS) with Sonazoid has a long-
\end{abstract}

Registered at Clinicaltrials.gov (NCT00822991).

Masatoshi Kudo and Kazuomi Ueshima contributed equally to this paper. 


\section{Liver Cancer}

lasting, stable Kupffer phase, which makes it possible to scan the entire liver to depict small lesions. In addition, defect reperfusion imaging (reinjection imaging) enables to determine whether the detected nodule is HCC or not. This prospective, multicenter, randomized, controlled trial was conducted to demonstrate the usefulness of Kupffer phase surveillance in the detection of small HCC compared to B-mode US. Methods: A total of 23 institutions joined this study. In total, 656 patients with hepatitis B- or C-related liver cirrhosis were randomized either to the B-mode US surveillance group $(n=313)$ or the Kupffer phase CEUS with Sonazoid surveillance group $(n=309)$. The primary endpoint was the maximum size of HCC at the time of the first detection. Secondary endpoints included time to HCC detection, number of tumors, and Barcelona Clinic Liver Cancer stage at the first detection, and sensitivity, specificity, and accuracy of each method in the diagnosis, and the cumulative detection rate of HCC. $\boldsymbol{R e}$ sults: The mean HCC size at the first detection was significantly smaller in the CEUS (13.0 \pm $4.1 \mathrm{~mm} ; n=28)$ than in the B-mode US group (16.7 $\pm 4.1 \mathrm{~mm} ; n=26)(p=0.011)$. Of the 38 patients with HCV cirrhosis diagnosed with HCC by US alone, mean tumor size at the first detection was significantly smaller in the 20 patients diagnosed by CEUS alone than in the 18 diagnosed by B-mode US alone (12.7 \pm 3.1 vs. $17.6 \pm 7.0 \mathrm{~mm}, p=0.012)$. In contrast, among the 16 patients with HBV cirrhosis diagnosed by US alone, mean tumor size at the first detection was similar in the 8 patients diagnosed by CEUS alone and the 8 diagnosed by B-mode US (13.6 \pm 6.0 vs. $14.5 \pm 2.7 \mathrm{~mm}, p=0.715$ ). Conclusion: Kupffer phase CEUS surveillance with Sonazoid is extremely useful for the early detection and confirmation of HCC using a reinjection technique. Kupffer phase CEUS with Sonazoid contrast combined with the reinjection technique is, therefore, recommended as first-line screening tool for HCC in patients with liver cirrhosis, especially those with very coarse liver parenchyma.

(c) 2019 S. Karger AG, Basel

\section{Introduction}

All current practice guidelines, including those of the American Association for the Study of Liver Diseases (AASLD), the European Association for the Study of the Liver (EASL), the Asian Pacific Association for the Study of the Liver (APASL), and the Japan Society of Hepatology (JSH), recommend the use of ultrasound (US) as an initial surveillance tool for hepatocellular carcinoma (HCC) in patients with liver cirrhosis [1-5]. Patients with liver cirrhosis, however, frequently have coarse liver parenchyma, which mask the presence of tiny nodules during B-mode US.

Sonazoid is a US contrast agent that provides superb real-time vascular imaging and long-lasting stable Kupffer phase imaging, lasting for more than $60 \mathrm{~min}$, and thus enables multiple scans of the entire liver [6]. The favorable features of Sonazoid allow its reinjection at the Kupffer phase and its use for surveillance of patients with liver cirrhosis [7, 8]. Sonazoid has been approved for use in Japan, Korea, Taiwan, China, and Singapore as an ultrasound contrast agent, but it has not yet been approved in Europe apart from Norway [9].

In the surveillance setting, the diagnostic criteria for HCC differ for B-mode US, contrastenhanced US (CEUS), and contrast-enhanced CT/MRI. The characteristics of HCC on B-mode US include a mosaic pattern, halo, and septum, whereas the characteristics of HCC on CEUS include a defect in the Kupffer phase 10-60 min after injection and arterial enhancement following reinjection of Sonazoid at the Kupffer phase. In comparison, the characteristics of HCC on contrast-enhanced CT/MRI include arterial enhancement with venous washout. Comparing CEUS with contrast-enhanced CT in patients with HCC, the characteristics of CEUS were found to be clearer than those of dynamic CT due to arterial enhancement with Kupffer defects [10]. 


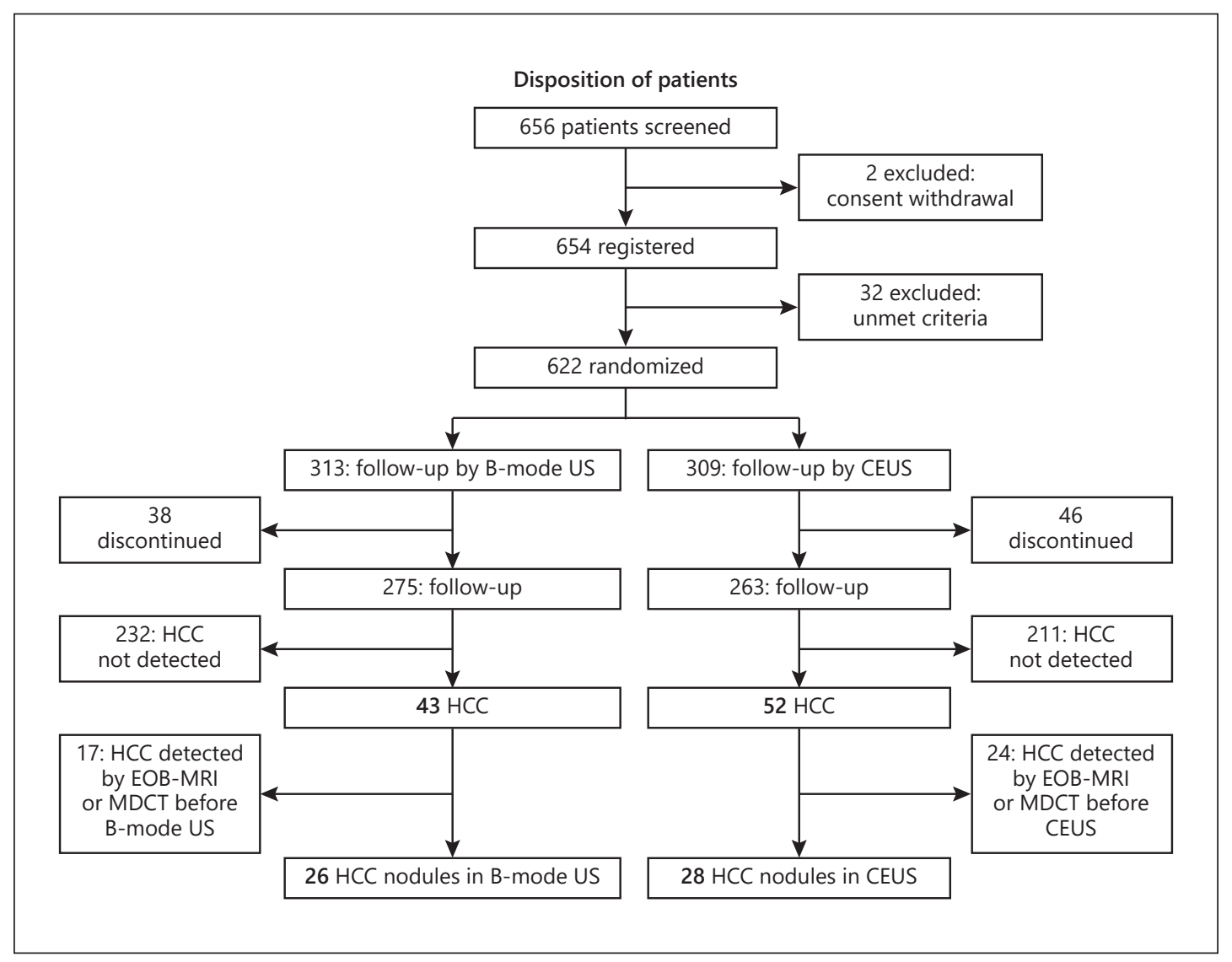

Fig. 1. Flow chart of the study protocol.

Although CT and MRI may be more specific than B-mode US in detecting HCC, the former methods are more expensive, especially for the surveillance of cirrhotic patients [11]. In addition, CT and MRI are regarded as diagnostic, not as screening, tools. For cirrhotic patients, $\mathrm{CT} / \mathrm{MRI}$ is covered by the insurance in Japan but not in other countries. The HCC practice guidelines developed by the JSH recommend that surveillance consists of US examination and tumor marker assessment (AFP, DCP, and AFP-L3) every 3-4 months and dynamic CT or MRI every 6-12 months $[3,5,12]$.

A previous study found that 16 of 292 (5\%) small hypervascular HCCs, ranging from 6-13 $\mathrm{mm}$ in diameter, which were not detected by B-mode US, were detected by Kupffer phase CEUS surveillance [13]. In addition, the rates of intrahepatic metastases and microvascular invasion after curative treatment are proportional to the diameter of the primary HCC [14]. Thus, Kupffer phase CEUS with Sonazoid may be a better surveillance tool than B-mode US for identifying small lesions. We, therefore, compared CEUS and B-mode US surveillance in cirrhotic patients at very high risk of developing HCC.

\section{Methods}

A total of 656 Japanese patients with HBV- or HCV-related liver cirrhosis considered at very high risk for HCC development were enrolled. Patients were included if they were aged > 20 years; had HBV- or HCVrelated liver cirrhosis (confirmed by liver biopsy or radiologically); portal hypertension or platelet count $<130,000 / \mathrm{mL}$; and no history of HCC; and if they provided informed consent. Patients were excluded if they 
Kudo et al.: B-Mode versus Contrast-Enhanced US for HCC Surveillance

Table 1. Baseline demographic and clinical characteristics of the CEUS and the B-mode US group

\begin{tabular}{|c|c|c|c|}
\hline Characteristics & $\begin{array}{l}\text { CEUS } \\
(n=309)\end{array}$ & $\begin{array}{l}\text { B-mode US } \\
(n=313)\end{array}$ & $p$ value \\
\hline \multicolumn{4}{|l|}{ Age, years } \\
\hline Median (range) & $68.0(61.0-75.0)$ & $67.0(58.0-74.0)$ & 0.200 \\
\hline Mean \pm SD & $66.9 \pm 10.2$ & $65.7 \pm 11.2$ & 0.152 \\
\hline Gender, males/females & $136 / 173$ & $132 / 181$ & 0.643 \\
\hline Etiology & & & 0.639 \\
\hline HBV (NA+) & 78 & 85 & \\
\hline HBV (NA-) & 23 & 18 & \\
\hline $\mathrm{HCV}$ & 208 & 210 & \\
\hline Median albumin (range), mg/dL & $3.9(3.6-4.3)$ & $4.0(3.6-4.3)$ & 0.676 \\
\hline Median bilirubin (range), mg/dL & $0.90(0.70-1.20)$ & $0.81(0.70-1.17)$ & 0.360 \\
\hline Median PT (range), INR & $1.09(1.02-1.17)$ & $1.08(1.01-1.16)$ & 0.247 \\
\hline Median PT (range), \% & $85.0(75.0-96.7)$ & $85.5(76.5-98.7)$ & 0.347 \\
\hline Encephalopathy & & & 0.684 \\
\hline None & 306 & 311 & \\
\hline Grades I, II & 3 & 2 & \\
\hline Grades III, IV & 0 & 0 & \\
\hline Ascites & & & 0.114 \\
\hline None & 293 & 285 & \\
\hline Mild & 15 & 27 & \\
\hline Moderate & 1 & 1 & \\
\hline Child-Pugh score & & & 0.826 \\
\hline 5 & 206 & 198 & \\
\hline 6 & 58 & 67 & \\
\hline 7 & 15 & 16 & \\
\hline 8 & 5 & 8 & \\
\hline 9 & 3 & 3 & \\
\hline 10 & 1 & 0 & \\
\hline Median AFP (range), ng/dL & $6.6(3.1-14.0)$ & $6.5(3.4-16.8)$ & 0.945 \\
\hline Median AFP-3 (range), \% & $1.6(0.0-6.2)$ & $1.2(0.0-5.1)$ & 0.597 \\
\hline Median PIVKA-II (DCP) (range), mAU/mL & $19.0(15.0-24.0)$ & $18.0(14.0-24.0)$ & 0.327 \\
\hline Median hyaluronic acid (range), ng/mL & $203(88-420)$ & $198(98-394)$ & 0.955 \\
\hline Median platelet count (range), $\times 10^{4} / \mu \mathrm{L}$ & $9.7(7.4-11.8)$ & $10.1(7.6-12.0)$ & 0.394 \\
\hline Median AST (range), IU/L & $44.0(31.0-61.0)$ & $41.0(28.0-59.5)$ & 0.126 \\
\hline Median ALT (range), IU/L & $35.0(23.5-50.0)$ & $33.0(22.0-52.5)$ & 0.226 \\
\hline
\end{tabular}

had a history of hypersensitivity to egg yolk, severe liver dysfunction (AST, ALT, or bilirubin >10 $\times$ ULN), cirrhosis associated with HCC, and treatment with interferon, and were aged $<20$ years or judged inappropriate for inclusion by the study investigator. In addition, women with confirmed or suspected pregnancy and lactating women were excluded.

Patients were randomized 1:1 to B-mode US $(n=313)$ or Kupffer phase CEUS $(n=309)$ surveillance. Patients were stratified into those with HCV cirrhosis, those with HBV cirrhosis treated with a nucleoside analogue, and those with HBV cirrhosis not treated with a nucleoside analogue. Surveillance consisted of B-mode US or Kupffer phase CEUS every $4 \pm 1$ months and CT/MRI every 8 months. Patients in the CEUS group were scanned during the Kupffer (postvascular) phase 10-40 min after Sonazoid injection. Only if the Kupffer defect is depicted, reinjection of Sonazoid was performed to confirm HCC.

\section{Study Endpoints}

The primary endpoint of the study was the maximum size of HCC at the first detection. Secondary endpoints included time to HCC detection; number of tumors, and Barcelona Clinic Liver Cancer (BCLC) stage at the first detection; ability to diagnose HCC; the sensitivity, specificity, and accuracy of diagnosis; and the cumulative rate of HCC detection. 


\section{Liver Cancer}

Fig. 2. a Tumor size at the first detection by B-mode US or Kupffer phase CEUS without EOB-MRI or MDCT in the cohort $(n=54)$. The maximum size of initially detected HCC nodules was significantly smaller in the surveillance arm with Kupffer phase CEUS with reinjection $(16.7 \pm 6.2 \mathrm{~mm}[9-32$ $\mathrm{mm}])$ than with the B-mode US $(13.0 \pm 4.1 \mathrm{~mm}[6-25 \mathrm{~mm}])(p=$ $0.011)$. b Tumor size at the first detection by hepatitis virus type. There was a significant difference in tumor size in HCV-related HCC $(p=0.012)$; however, no significant difference was observed in HBV-related HCC ( $p=0.715)$.

\begin{tabular}{|c|c|}
\hline \multicolumn{2}{|l|}{ Liver Cancer 2019;8:271-280 } \\
\hline DOI: 10.1159/000501082 & $\begin{array}{l}\text { (c) } 2019 \text { S. Karger AG, Basel } \\
\text { www.karger.com/lic }\end{array}$ \\
\hline
\end{tabular}

Kudo et al.: B-Mode versus Contrast-Enhanced US for HCC Surveillance
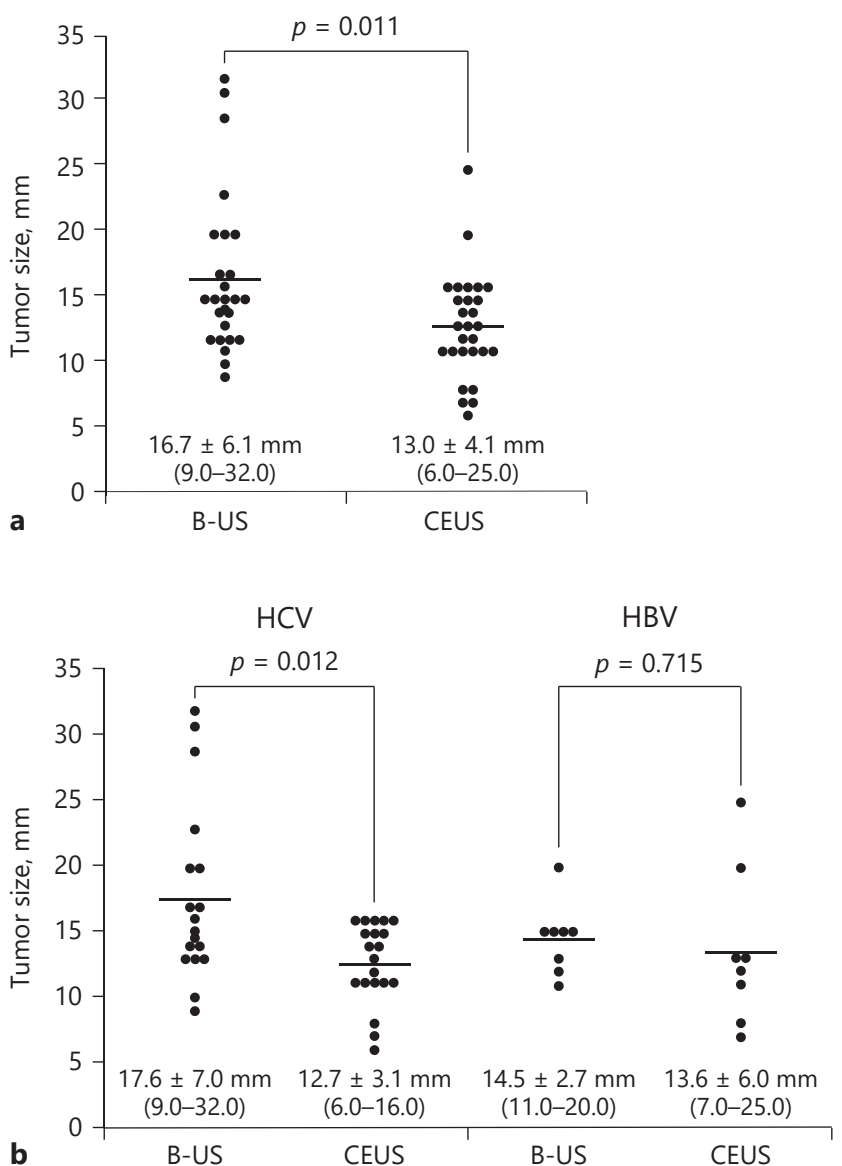

\section{Statistical Methods}

Significant differences in tumor size between the CEUS and the B-mode US group at the time of HCC detection were assessed by determining whether the sizes were normally distributed. Normally distributed data were compared by Student's or Welch's $t$ test, whereas nonnormally distributed data were compared using the Mann-Whitney $U$ test. The sensitivity, specificity, and accuracy of the diagnosis and the cumulative rate of HCC detection were analyzed.

The time from study start to tumor detection in the CEUS and the B-mode US group was assessed using the Kaplan-Meier method and compared by log-rank test. Tumor numbers and stage at the first HCC detection were compared using the Mann-Whitney U test. Patient overall survival was assessed using the Kaplan-Meier method and compared using the same log-rank test.

\section{Results}

\section{Patient Disposition}

Of the 656 patients randomized, 203 (30.9\%) had HBV- and 453 (69.1) had HCV-related cirrhosis. Patient disposition is shown in Figure 1. Baseline characteristics of the B-mode US and the CEUS group are shown in Table 1 . The two groups were well matched with no significant differences in any baseline clinical or demographic characteristic. 


\section{Liver \\ Cancer}

Table 2. Characteristics of HCC at first detection

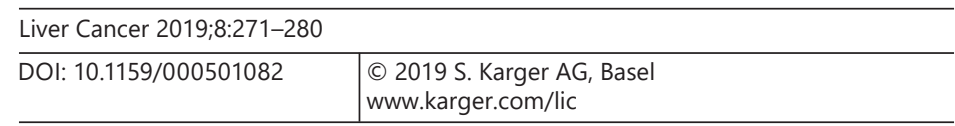

Fig. 3. Annual HCC incidence was $5.5 \%$ in HBV-related cirrhotic patients and $7.1 \%$ in HCV-related cirrhotic patients.

\begin{tabular}{ccc}
\hline & $\begin{array}{l}\text { B-mode US } \\
(n=26)\end{array}$ & $\begin{array}{l}\text { CEUS } \\
(n=28)\end{array}$ \\
\hline $\begin{array}{c}\text { Child-Pugh grade } \\
\text { A }\end{array}$ & $21(81 \%)$ & $17(61 \%)$ \\
B & $4(15 \%)$ & $11(39 \%)$ \\
C & $1(4 \%)$ & $0(0 \%)$ \\
BCLC stage & $17(65 \%)$ & $24(86 \%)$ \\
0 & $7(27 \%)$ & $4(14 \%)$ \\
A & $1(4 \%)$ & $0(0 \%)$ \\
B & $1(4 \%)$ & $0(0 \%)$ \\
D & & \\
\hline
\end{tabular}

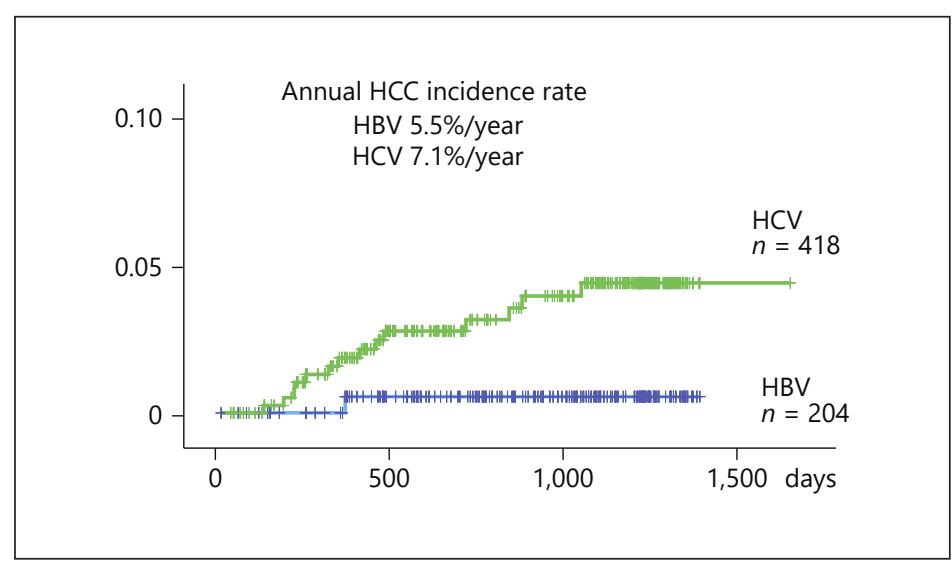

\section{Diagnosis of HCC}

Of the 656 patients, 95 (14.5\%) were diagnosed with HCC during the study period, including 43 diagnosed in the B-mode US surveillance group and 52 in the CEUS surveillance group; 41 patients were diagnosed by CT or MRI before B-mode US or CEUS was performed. Therefore, in order to exclude potential bias, these 41 patients were excluded from the final analysis. Of the 26 patients diagnosed by B-mode US alone, 18 had HCV- and 8 had HBVrelated cirrhosis; however, of the 28 patients diagnosed by CEUS alone, 20 had HCV- and 8 had HBV-related cirrhosis. Mean examination time for B-mode US was $16.2 \mathrm{~min}$, whereas mean examination time for a Kupffer phase scan was $6.6 \mathrm{~min}$. When reinjection was performed for lesions showing the Kupffer defect, mean examination time was $12.3 \mathrm{~min}$.

\section{Assessment of Primary Endpoints}

Mean tumor size at the first detection in the B-mode US and the CEUS group showed that HCC size was significantly smaller in the CEUS group $(13.0 \pm 4.1 \mathrm{~mm} ; n=28)$ than in the B-mode US group (16.7 $\pm 4.1 \mathrm{~mm} ; n=26$; Fig. $2 \mathrm{a})$ ( $\mathrm{p}=0.011$ ). Of the 38 patients with HCV cirrhosis diagnosed with HCC by US alone, mean tumor size at the first detection was significantly smaller in the 20 patients diagnosed by CEUS alone $(12.7 \pm 3.1 \mathrm{~mm})$ than in the 18 diagnosed by B-mode US alone (17.6 $\pm 7.0 \mathrm{~mm} ; p=0.012$; Fig. $2 \mathrm{~b})$. In contrast, among the 16 patients with HBV cirrhosis diagnosed by US alone, mean tumor size at the first detection was similar in the 8 patients diagnosed by CEUS alone $(13.6 \pm 6.0 \mathrm{~mm})$ and the 8 diagnosed by B-mode US (14.5 $\pm 2.7 \mathrm{~mm} ; p=0.715)$. 


\section{Liver Cancer}

\begin{tabular}{l|l|}
\hline Liver Cancer 2019;8:271-280 \\
\hline DOI: 10.1159/000501082 & $\begin{array}{l}\text { (c) 2019 S. Karger AG, Basel } \\
\text { www.karger.com/lic }\end{array}$ \\
\hline
\end{tabular}

Kudo et al.: B-Mode versus Contrast-Enhanced US for HCC Surveillance

Table 3. Treatment at first HCC detection by modality

\begin{tabular}{lcccc}
\hline Modality & $\begin{array}{l}\text { Total } \\
(n=95)\end{array}$ & $\begin{array}{l}\text { CT/MRI } \\
(n=41)\end{array}$ & $\begin{array}{l}\text { B-mode US } \\
(n=26)\end{array}$ & $\begin{array}{l}\text { CEUS } \\
(n=28)\end{array}$ \\
\hline Ablation $^{\mathrm{a}}$ & $62(65.3 \%)$ & $25(61.0 \%)$ & $17(65.4 \%)$ & $20(71.4 \%)$ \\
Resection $_{\text {TACE }}^{21(22.1 \%)}$ & $11(26.8 \%)$ & $5(19.2 \%)$ & $5(17.9 \%)$ \\
Others & $8(8.4 \%)$ & $4(9.8 \%)$ & $3(11.5 \%)$ & $1(3.6 \%)$ \\
& $4(4.2 \%)$ & $1(2.4 \%)^{\mathrm{b}}$ & $1(3.8 \%)^{\mathrm{c}}$ & $2(7.1 \%)^{\mathrm{d}}$ \\
\hline
\end{tabular}

a All underwent radiofrequency ablation, except for 8 patients in the CEUS group. ${ }^{b}$ Deterioration of liver function. ${ }^{\mathrm{c}}$ Refused treatment. ${ }^{\mathrm{d}}$ One each refused treatment and showed deterioration of liver function.

Table 4. Detectability and diagnostic ability

\begin{tabular}{|c|c|c|c|c|}
\hline & \multicolumn{2}{|c|}{ CEUS $(n=309)$} & \multicolumn{2}{|c|}{ B-mode US $(n=313)$} \\
\hline & HCC & no HCC & HCC & no HCC \\
\hline Positive & 28 & 11 & 17 & 9 \\
\hline Negative & 0 & 270 & 9 & 278 \\
\hline Sensitivity & \multicolumn{2}{|c|}{$28 / 28$ (100\%) } & \multicolumn{2}{|c|}{$17 / 26 \quad(65.4 \%)$} \\
\hline Specificity & \multicolumn{2}{|c|}{$270 / 281(96.1 \%)$} & \multicolumn{2}{|c|}{$278 / 287$ (96.9\%) } \\
\hline Accuracy & \multicolumn{2}{|c|}{$298 / 309(96.4 \%)$} & \multicolumn{2}{|c|}{$295 / 313(94.2 \%)$} \\
\hline PPV & \multicolumn{2}{|c|}{ 28/39 (71.8\%) } & \multicolumn{2}{|c|}{$17 / 26 \quad(65.4 \%)$} \\
\hline NPV & \multicolumn{2}{|c|}{$270 / 270(100 \%)$} & \multicolumn{2}{|c|}{$278 / 287(96.9 \%)$} \\
\hline
\end{tabular}

Secondary Endpoints

Of the 26 patients diagnosed with HCC by B-mode US alone, 24 had 1 nodule and 1 patient each had 2 and 3 HCC nodules at the time of diagnosis. In comparison, of the 28 patients diagnosed with HCC by CEUS, 25 had 1 nodule, 2 had 2 nodules, and 1 had 3 nodules at the time of diagnosis. Child-Pugh grade and BCLC stage at the first detection of HCC were similar in the B-mode US and the CEUS group (Table 2). The annual incidence rates of HCC were $5.5 \%$ per year in patients with HBV cirrhosis and $7.1 \%$ per year in patients with HCV cirrhosis (Fig. 3).

At a median follow-up period of 2.97 years ( $95 \% \mathrm{CI}, 2.85-3.08$ years), the median times to the first $\mathrm{HCC}$ detection were 4.17 years ( $95 \% \mathrm{CI}, 4.05-4.29$ years) for B-mode US and 3.51 years (95\% CI, 3.41-3.61 years) for CEUS ( $p=0.786)$. At the same median follow-up period, median overall survival was 4.41 years ( $95 \% \mathrm{CI}, 4.34-4.48$ years) for patients in the B-mode US group alone and 3.72 years (95\% CI, 3.41-3.77 years) for patients in the CEUS group alone $(p=0.785)$. Initial treatments at the first detection of HCC were similar in patients diagnosed by CT/MRI, B-mode US, and CEUS (Table 3 ).

During the surveillance period, 19 of the 656 patients died, 4 of liver failure; 4 of pneumonia; 2 from traffic accidents; 2 of spontaneous bacterial peritonitis; 1 each of cerebral infarction, lung cancer, rupture of esophageal varices, sepsis of unknown cause, and malignant lymphoma, and 2 of unknown causes. There were no protocol-related deaths or deaths from HCC.

The sensitivity, specificity, accuracy, and positive and negative predictive values for the detection of HCC were 100, 96.1, 96.4, 71.8, and 100\%, respectively, for CEUS, compared with 65.4, 96.9, 94.2, 65.4, and 96.9\%, respectively, for B-mode US. The gold standard for sensitivity/specificity was set as hallmark findings by dynamic CT/MRI at the cutoff point. However, 


\section{Liver

Kudo et al.: B-Mode versus Contrast-Enhanced US for HCC Surveillance

further observation after the cutoff point in 11 patients without HCC diagnosis by CT/MRI, due to atypical finding by these modalities, showed typical findings of HCC in all patients, suggesting that CEUS has superior diagnostic ability than dynamic CT/MRI for small HCC nodules (sensitivity 100\%, specificity 100\%, accuracy 100\%) after all (Table 4).

\section{Safety Findings}

One patient experienced a grade 1 rash attributed to Sonazoid. Otherwise, no adverse event related with Sonazoid was observed. No deaths or severe adverse events were attributed to Sonazoid. Moreover, there were no HCC-related deaths throughout this study.

\section{Discussion}

This prospective randomized clinical trial comparing B-mode US and Kupffer phase CEUS surveillance of patients with liver cirrhosis for HCC demonstrated that Kupffer phase CEUS with Sonazoid contrast agent detected significantly smaller HCC nodules than B-mode US. These findings were both statistically significant and clinically meaningful. In Japan, the clinical practice guideline recommends surveillance with US and 3 tumor markers (AFP, DCP, and AFP-L3) every 3-4 months for HBV-related- and HCV-related cirrhotic patients [3, 5]. In addition, the guideline also recommends surveillance of super-high-risk HCC patients by EOB-MRI or dynamic CT every 6-12 months. Therefore, in this study, main analysis was performed in HCC patients initially detected by B-mode US or Kupffer phase CEUS with Sonazoid alone. In other words, HCCs detected by EOB-MRI or dynamic CT before B-mode US or CEUS surveillance were excluded from the final analysis because of the potential bias. However, since this is not the case in other countries, analysis in the present study might have been too strict.

When we confined the data to HCCs not detected by EOB-MRI or MDCT before B-mode US or CEUS surveillance, all HCCs detected by Kupffer phase CEUS were either BCLC stage 0 $(86 \%)$ or A $(14 \%)$. In comparison, $92 \%$ of HCCs detected by B-mode US were BCLC stage 0 $(65 \%)$ or A (27\%), suggesting that CEUS detects HCCs at earlier stages than B-mode US.

Of the 28 patients with HCC detected by CEUS, $89 \%$ received curative treatments such as ablation $(71 \%)$ or resection (18\%). In comparison, $84 \%$ of patients with HCC detected by B-mode US received curative treatments, such as ablation (65\%) or resection (19\%).

Although 16 patients died during the study, no HCC-related death was observed. Most Japanese patients with cirrhosis, however, are elderly, with many dying of non-HCC-related causes. At a median follow-up of 3.0 years, overall survival and time to first HCC detection were similar in the B-mode US and the CEUS groups.

HCC can be confirmed earlier by Kupffer phase CEUS surveillance with Sonazoid reinjection technique $[7,8]$ than by dynamic CT/MRI since arterial enhancement with Kupffer defect, a typical hallmark of CEUS for small overt HCC nodules, can be observed much earlier due to the superb technique of the reinjection method $[7,8]$. This hallmark is more precise than those of dynamic CT/MRI. However, some small nodules not detected by B-mode US, due to coarse liver parenchyma, or CEUS, due to deeply seated HCC or HCC at the subphrenic region, may have been detected by CT or EOB-MRI easily.

Of note, only in Japan extensive surveillance with EOB-MRI or MDCT every 6-12 months is being conducted. Therefore, the mean size of all 95 HCCs detected either by EOB-MRI, dynamic CT, B-mode US, or CEUS was much smaller (data are not shown). These findings may be useful for other countries than Japan, where MDCT or EOB-MRI surveillance are not routinely performed.

In conclusion, Kupffer phase CEUS surveillance with Sonazoid reinjection is extremely useful in the early detection and confirmation of HCC with the reinjection technique. In 
addition, Kupffer phase CEUS surveillance is much easier, less stressful, and less time consuming ( $<7 \mathrm{~min}$ ) for the examiner than B-mode US ( $>15 \mathrm{~min}$ ) because the examiner only has to detect a black hole on the background white liver by CEUS with Sonazoid. Kupffer phase CEUS with Sonazoid contrast combined with the reinjection technique is, therefore, recommended as a first-line screening tool for HCC in patients with liver cirrhosis, especially those with very coarse liver parenchyma.

\section{Statement of Ethics}

The study protocol was approved by ethics committee or institutional review board at each participating institution, and written informed consent was obtained from all patients prior to enrolment. This trial was registered at Clinicaltrials.gov (NCT008229919). The study conformed to the Declaration of Helsinki.

\section{Disclosure Statement}

Masatoshi Kudo received honoraria from Daiichi-Sankyo and GE HealthCare; the other authors have no conflict of interest.

\section{Author Contributions}

Conception and design: Masatoshi Kudo and Kazuomi Ueshima; collection and assembly of data: Yukio Osaki, Masashi Hirooka, Yasuharu Imai, Kazunobu Aso, Kazushi Numata, Masayuki Kitano, Takashi Kumada, Namiki Izumi, Yasukiyo Sumino, and Chikara Ogawa; data analysis and interpretation: Masatoshi Kudo, Kazuomi Ueshima, and Kohei Akazawa; statistical analysis: Kohei Akazawa; writing and final approval of the manuscript: all authors.

\section{References}

1 Galle PR, Forner A, Llovet JM, Mazzaferro V, Piscaglia F, Raoul JL, et al.; European Association for the Study of the Liver. EASL clinical practice guidelines: Management of hepatocellular carcinoma. J Hepatol. 2018 Jul; 69(1):182-236.

2 Marrero JA, Kulik LM, Sirlin CB, Zhu AX, Finn RS, Abecassis MM, et al. Diagnosis, Staging, and Management of Hepatocellular Carcinoma: 2018 Practice Guidance by the American Association for the Study of Liver Diseases. Hepatology. 2018 Aug;68(2):723-50.

3 Kokudo N, Hasegawa K, Akahane M, Igaki H, Izumi N, Ichida T, et al. Evidence-based Clinical Practice Guidelines for Hepatocellular Carcinoma: The Japan Society of Hepatology 2013 update (3rd JSH-HCC Guidelines). Hepatol Res. 2015 Jan;45(2):123-7.

4 Omata M, Cheng AL, Kokudo N, Kudo M, Lee JM, Jia J, et al. Asia-Pacific clinical practice guidelines on the management of hepatocellular carcinoma: a 2017 update. Hepatol Int. 2017 Jul;11(4):317-70.

5 Kudo M, Matsui O, Izumi N, Iijima H, Kadoya M, Imai Y, et al.; Liver Cancer Study Group of Japan. JSH ConsensusBased Clinical Practice Guidelines for the Management of Hepatocellular Carcinoma: 2014 Update by the Liver Cancer Study Group of Japan. Liver Cancer. 2014 Oct;3(3-4):458-68.

6 Hatanaka K, Kudo M, Minami Y, Ueda T, Tatsumi C, Kitai S, et al. Differential diagnosis of hepatic tumors: value of contrast-enhanced harmonic sonography using the newly developed contrast agent, Sonazoid. Intervirology. 2008;51 Suppl 1:61-9.

7 Kudo M, Hatanaka K, Maekawa K. Newly developed novel ultrasound technique, defect reperfusion ultrasound imaging, using sonazoid in the management of hepatocellular carcinoma. Oncology. 2010 Jul; 78 Suppl 1:40-5.

8 Kudo M. Defect Reperfusion Imaging with Sonazoid ${ }^{\circledR}$ : A Breakthrough in Hepatocellular Carcinoma. Liver Cancer. 2016 Feb;5(1):1-7.

9 Claudon M, Dietrich CF, Choi BI, Cosgrove DO, Kudo M, Nolsøe CP, et al.; World Federation for Ultrasound in Medicine; European Federation of Societies for Ultrasound. Guidelines and good clinical practice recommendations for Contrast Enhanced Ultrasound (CEUS) in the liver - update 2012: A WFUMB-EFSUMB initiative in cooperation with representatives of AFSUMB, AIUM, ASUM, FLAUS and ICUS. Ultrasound Med Biol. 2013 Feb; 39(2):187-210. 
10 Hatanaka K, Kudo M, Minami Y, Maekawa K. Sonazoid-enhanced ultrasonography for diagnosis of hepatic malignancies: comparison with contrast-enhanced CT. Oncology. 2008;75 Suppl 1:42-7.

11 Ahmed Mohammed HA, Yang JD, Giama NH, Choi J, Ali HM, Mara KC, et al. Factors Influencing Surveillance for Hepatocellular Carcinoma in Patients with Liver Cirrhosis. Liver Cancer. 2017 Feb;6(2):126-36.

12 Kudo M. Management of Hepatocellular Carcinoma in Japan as a World-Leading Model. Liver Cancer. 2018 May;7(2):134-47.

13 Kudo M, Hatanaka K, Kumada T, Toyoda H, Tada T. Double-contrast ultrasound: a novel surveillance tool for hepatocellular carcinoma. Am J Gastroenterol. 2011 Feb;106(2):368-70.

14 Nakashima O, Sugihara S, Kage M, Kojiro M. Pathomorphologic characteristics of small hepatocellular carcinoma: a special reference to small hepatocellular carcinoma with indistinct margins. Hepatology. 1995 Jul;22(1):101-5. 